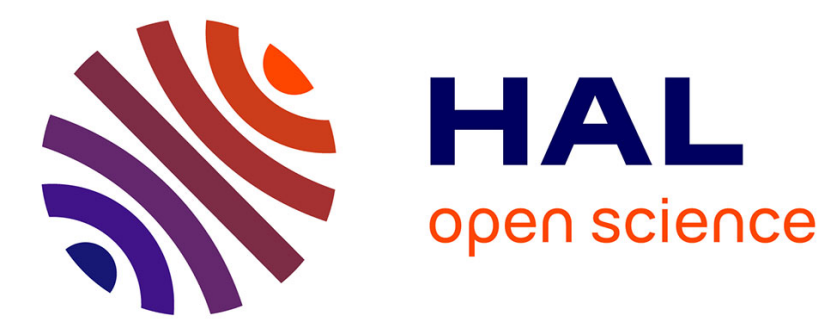

\title{
Geographic routing protocol for the deployment of virtual power plant within the smart grid
}

\author{
Mouna Rekik, Zied Chtourou, Nathalie Mitton, Ahmad Atieh
}

\section{To cite this version:}

Mouna Rekik, Zied Chtourou, Nathalie Mitton, Ahmad Atieh. Geographic routing protocol for the deployment of virtual power plant within the smart grid. Sustainable Cities and Society, 2016, 10.1016/j.scs.2015.12.003 . hal-01252686

\section{HAL Id: hal-01252686 \\ https://inria.hal.science/hal-01252686}

Submitted on 8 Mar 2016

HAL is a multi-disciplinary open access archive for the deposit and dissemination of scientific research documents, whether they are published or not. The documents may come from teaching and research institutions in France or abroad, or from public or private research centers.
L'archive ouverte pluridisciplinaire HAL, est destinée au dépôt et à la diffusion de documents scientifiques de niveau recherche, publiés ou non, émanant des établissements d'enseignement et de recherche français ou étrangers, des laboratoires publics ou privés. 


\title{
Geographic routing protocol for the deployment of virtual power plant within the smart grid
}

\author{
Mouna Rekik ${ }^{\mathrm{a}, \mathrm{b}}$, Zied Chtourou ${ }^{\mathrm{a}}$, Nathalie Mitton ${ }^{\mathrm{b}}$, Ahmad Atieh $^{\mathrm{c}}$ \\ ${ }^{a}$ CMERP, Sfax, Tunisia. \\ ${ }^{b}$ Inria Lille - Nord Europe, France. \\ ${ }^{c}$ The University of Jordan, Amman 11942 Jordan
}

\begin{abstract}
Smart grid (SG) incorporates communication networks to conventional electricity system in order to intelligently integrate distributed energy resources (DERs) and to allow demand side management. The move to SG in developing countries has to cope with great disparities of information and communication technology (ICT) infrastructures even within the same city. Besides, individual DERs are often too small to be allowed access to the energy market. Likewise power utilities are unable to effectively control and manage small DERs. An affordable and scalable wireless communication technology is proposed to aggregate geographically sparse DERs into a single virtual power plant (VPP). An ant colony optimization (ACO) method is used for the first time to the best of our knowledge in conjunction with geographic greedy forwarding protocol to effectively deliver data packets without void problems for VPP. The VPP investigated is dynamic and expected to scale up as more and more prosumers are attracted to join it. The proposed communication network and protocol follow the progression of the VPP to allow scalability and rapid deployment. The routing algorithm used for data communication within the VPP supports centralized, decentralized or fully distributed controlled VPP's DERs.
\end{abstract}

Keywords: Smart Grid, Virtual Power Plant, Point-to-Point Traffic, Wireless Sensor Network, Routing Protocol, Geographic Routing

Email addresses: mouna.rekik@mail.com (Mouna Rekik), ziedchtourou@gmail.com (Zied Chtourou), nathalie.mitton@inria.fr (Nathalie Mitton), a.atieh@ju.edu.jo (Ahmad Atieh) 


\section{Introduction}

The electric grids are undergoing radical changes to transform into SG promoting sustainable energy resources and reducing carbon emissions resulting from energy generation and consumption. Conventional electricity grid has been developed based on the assumption that electricity is generated at few stations generally outside urban areas and transported and distributed to large, medium and small sized consumers. The grid is evolving to a power network with distributed energy resources and storages (DERSs) combined with large central power plants. Electricity can be generated, consumed and stored anywhere in the grid. Renewable energy sources offer pollution free, climate friendly, sustainable and unlimited sources of energy. Their integration into the power grid is driven largely by environmental and economic regulation aimed at transforming into a clean and sustainable energy grid. Smart governments promote deployment of "in-grid" technologies that are susceptible to improve operational efficiency and labor use and reduce capital investment through management of peak demand. Indeed, SG technologies allow for better prediction of electricity supply and demand anywhere in the grid and provide efficient grid conditions monitoring and control. Moreover, it will give energy prosumers provisions to actively interact in managing demand and/or production on their side and to contribute to the resilience and efficiency of the electricity system through responsible interaction with the grid state. In fact, instead of continuously rushing to satisfy customer demand, SG integrates demand-side management (DSM) programs that aim at adapting demand to available production. These strategies try to fulfill load profile constraints either through providing financial incentives to consumers to encourage them to shift their energy demand to periods with extra available power, or through direct control of energy intensive devices like air conditioners and water heaters [1]. The effectiveness of these strategies is somewhat relativistic. It has been found that these strategies can certainly shift part of the load demand, but, conversely, could lead to new demand peaks as soon as the DSM signal is off and introduce artificial periodicity in the load profile [2].

The integration of DERSs and DSM programs is a key, yet challenging, feature for SG implementation. An information communication networks is deployed in parallel to the power network to coordinate bidirectional power

flows and support related services and actions in order to accommodate high integration of sustainable energy systems and enable development of innova- 
tive smart sustainable energy programs.

As mentioned earlier, the integration of DERSs into the grid, in particular renewable energy sources or natural storage, act for a sustainable energy system with less environmental concerns, more diversified energy resources and enhanced energy efficiency. However, DERS units working isolated from each other because of their different ownerships. They help satisfying local needs for a house, a building or a business but their integration within a grid could rapidly become a headache for utilities and distribution system operators (DSOs) for their influence on grid's stability, voltage levels, power factor, etc. The existing approaches for DERS integration are divided in two main classes based on prosumer participation and management strategies [3]: individual integration, and group integration.

Individual Integration strategy consists of connecting the prosumers directly to the grid. This facilitates direct prosumer-utility interactions. However, due to their small size, DERs are invisible to the grid as well as to the electricity market. Moreover, the number of DERs is expected to rise quickly, and with the variable amount of generated energy, the uncertainty need to be addressed in real time through active grid management. This integration strategy is clearly unsustainable.

Group Integration strategy consists of grouping a number of prosumers, with different energy resources and storage who are having diverse behaviors in consuming/producing energy, in a single entity and identifying them as Virtual Power Plant (VPP) to the utilities or DSO. A VPP knits together DERs including power generators, controllable loads and storage as a single generator. Any residential energy user or producer can be hooked up to the VPP. Energy that is generated but not used can also contribute to the VPP's capacity. The group of prosumers can attain the critical amount of energy, load and storage requested by utility companies to take part in energy auctions or be considered for ancillary services. Thus, the group integration seems more feasible, scalable and sustainable strategy.

In this work, we investigated an information and technology protocol to support VPP deployment. We analyzed data communication requirements for VPPs in terms of latency, delivery rate and communication types. Also, we provided data routing protocol that satisfies VPP requirements. The proposed protocol is scalable and capable of following VPP deployment at the different stages. It can handle different communication types among participants of the VPP such as multipoint-to-point, point-to-multipoint, and point-to-point. The proposed protocol can also accommodate VPP dynamic 
structure with seamless enrollment and dis-enrollment of prosumers. The protocol is capable of finding optimal communication paths with small number of control packets. The newly proposed routing protocol uses geographic routing (GR) combined with ACO concept in order to solve the communication void problem in GR. The main reason behind using ACO is to explore reliable paths around void areas. In fact wireless sensor networks suffer from such a problem in urban environment that have very dynamic structure due to radio frequency propagation and inherent fading. Therefore, an available node at a specific time is likely to become unavailable for relaying packets some milliseconds after. Hence, it's not useful to spend resources on optimization to find optimal paths each time we reach a void area. Indeed, a node within new path could itself become unavailable because of RF propagation. The performance of the overall protocol is shown to be within the requirements of SG communication.

In the next section we introduce the concept of VPP and its control types. This will allow us to highlight communication needs for the VPP. Then we report routing protocol for low power and lossy networks (RPL). This protocol has been standardized by the internet engineering task force (IETF) and is intended for wide variety of applications including home automation, urban sensor networks and advanced metering infrastructure (AMI) systems[4]. We overview the RPL protocol with the objective of pointing limitations that can hinder its application in the VPP. Finally, numerical simulations are conducted and presented for the proposed protocol for different node densities of VPP.

\section{Virtual Power Plant (VPP)}

A VPP is a set of geographically sparse DERs including power generators, controllable loads and storages that are aggregated in a way to perform as a single power facility, as illustrated in Fig. 1. A set of units among the available DERs (home energy management systems (HEMSs), energy storage systems (ESSs), distributed generation (DG), electric vehicles (EVs)), surrounded with red circles, decided to participate into the VPP.The VPP management system consists of a technical VPP (TVPP) and a commercial VPP (CVPP). From a technical perspective, the TVPP tries to optimize control and coordination of DERs, as well as system operation. It interacts directly with the Distribution System Operator (DSO) providing him with a unique profile of the whole aggregated plant to assist with network man- 


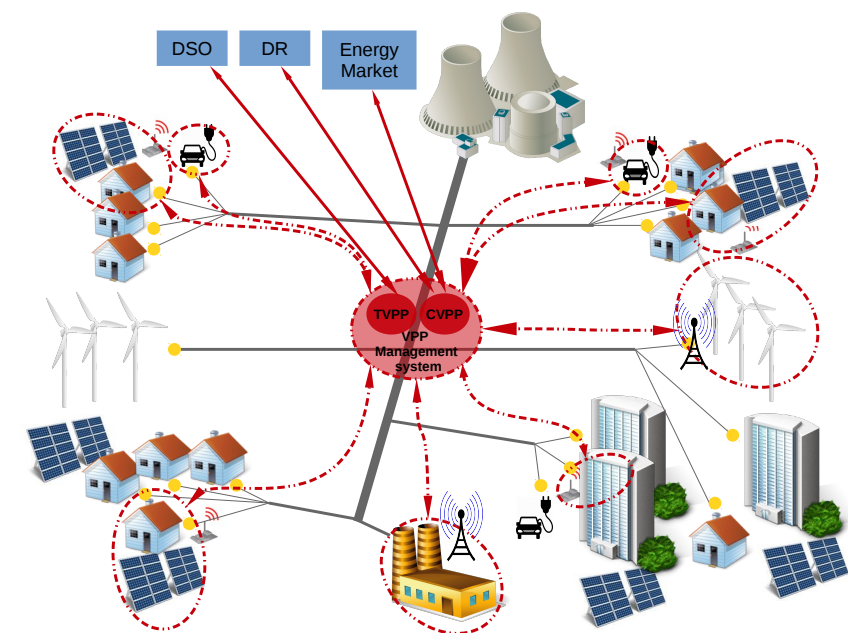

Fig. 1: Illustration of a VPP. The entities of the grid surrounded in red circles decided to join the VPP

agement and provide ancillary services. From an economical point of view, CVPP describes an economic aggregation of geographically sparse industrial, commercial and residential generation and consumption assets into a single business entity. It integrates demand response (DR) programs and DSM in order to trade the produced energy and/or shedded load to the best of owners interest. Hence, the CVPP directly interacts with the energy market.

\subsection{VPP business model}

A business model establishes the content, structure and governance of transactions designed to create value through the exploitation of business opportunities [5].

The VPP business model defines the bidding strategies into Independent System Operator (ISO)/wholesale markets while maximizing benefits for all VPP parties (utility, VPP operator, VPP participants).

According to [6], a set of factors need to be fixed in order to define an efficient business model for VPP: the method of financing (market or incentivized), the target market (system services, imbalance management, day ahead, intra-day, balancing market, etc.), the motivation factor (price structure, environmental aspect, system aspect, etc.), the customer type (household, commercial, industrial, public), the consumption characteristics (responsiveness, capacity, reliability, frequency, duration, etc.), the distributed 
generation characteristics (primary resource, responsiveness, capacity, reliability, frequency, duration, etc.), the activation type (response time, duration, changes, capacity, etc.), and the mode of communication or activation (manual, semi-automatic, automatic).

Several research papers proposed business models and strategies in order to optimize benefits of VPP and overcome the challenges that could be faced. A segmentation strategy for grouping customers based on location and type is proposed in [7]. In other words, the utility will have to manage several VPPs in order to provide better forecast and analytical information about the value participants could bring to the utility and thus achieve optimal benefits of VPPs. Ref. [8] and Ref. [9] address the problems faced by the VPP to participate in a joint market of energy and spinning reserve service and propose a bidding strategy that takes the supply-demand balancing constraint and security constraints of VPP itself into account.

The work in [10] proposes a truthful and reliable estimation approach and payment mechanism encouraging VPP managers and prosumers to form larger cooperative VPPs which minimizes the number of entities to interact with the grid and also to promote supply reliability.

In [11], the authors propose the use of VPP formed by wind power genera-

tors with EVs as an energy storage to overcome the intermittent nature of the energy supply. They define an agent-based approach that models VPP participation in electricity markets. They also introduce a payment scheme to compensate EVs for the use of their batteries.

The penetration of small distributed energy generation and storage systems into the power grid is expected to highly improve the sustainability of the power grid. However, distributed generation and storage systems are highly dynamic and depend mainly on prosumers behavior, which makes these systems very challenging for defining a business model. Hence, advanced control strategies and management approaches need to be applied in order to achieve an efficient and a reliable integration of small generation and energy storage systems into the grid.

\subsection{VPP Control Strategies}

Control of VPPs can be centralized, decentralized and fully distributed. In the centralized control [12], presented in Fig. 2, all knowledge about the DERs production and the energy market is located in the central controller. This gives the VPP a simple way of using DERs to meet grid demand. However, different ownership, and large number of DERs within a VPP challenge 
the central controller to find optimal control strategy in order to respond to best prosumers needs.

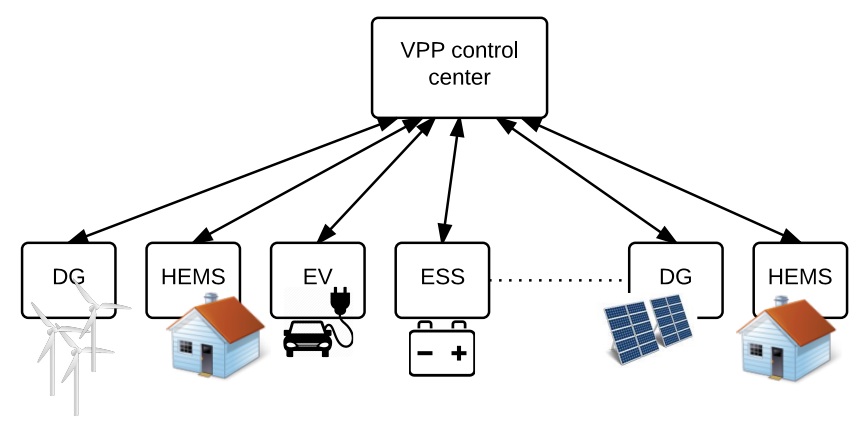

Fig. 2: Centralized controlled VPP

In the distributed control, the complexity is divided vertically within the VPP, as shown in Fig. 3. A hierarchical model is introduced in [13], by defining VPPs on different levels. A local VPP supervises and coordinates a limited number of DERs while delegating certain decisions upwards to a higher level VPP. This design requires communication between different neighboring VPPs.

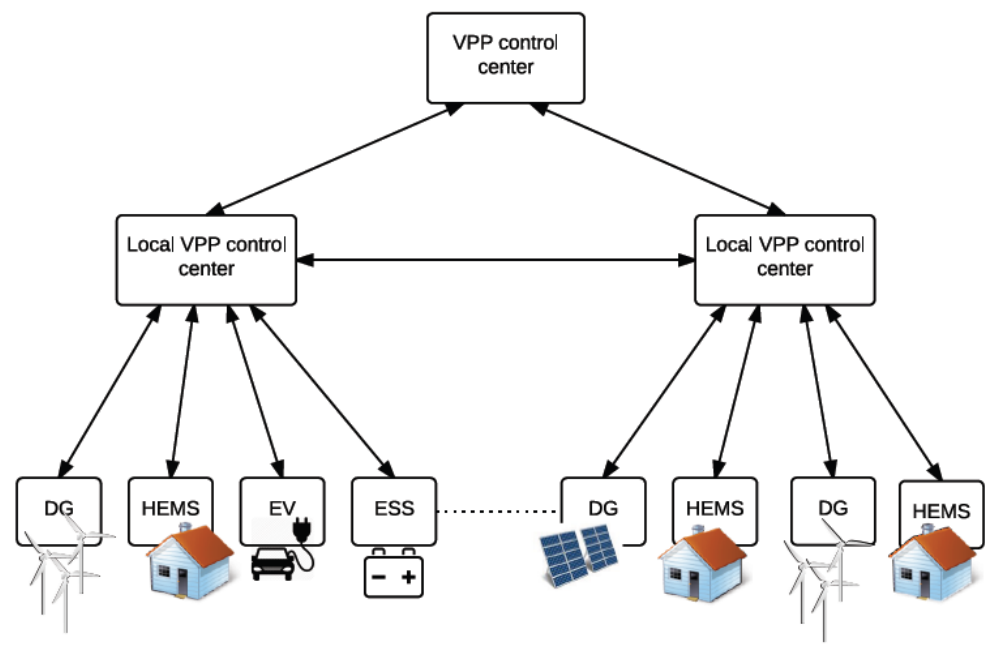

Fig. 3: Distributed controlled VPP: hierarchical model 
In the fully distributed control, each DER has its own communication and processing provisions in order to participate (and react) independently and smartly in the state of the power system. An example of such control is reported in [14] where minimal coordination between neighboring DERs is reported to be enough to reach global optimum state for the whole VPP. The work in [15] argues the use of decentralized control by the scale and

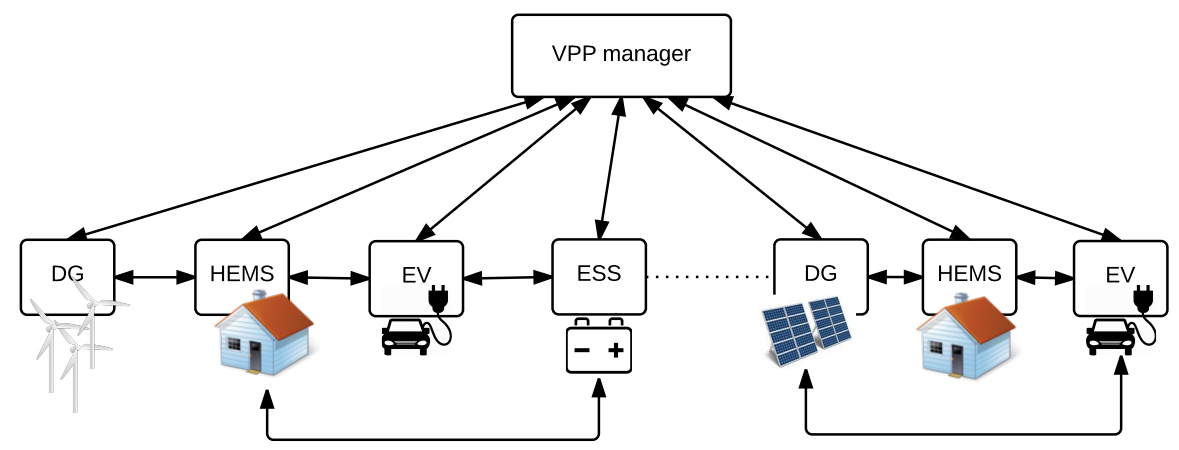

Fig. 4: Fully distributed controlled VPP

dynamism of this optimization problem of VPP. In fact, participants of VPP have a highly dynamic nature, some produce energy at specific times of the day, VPP partners need, then, to be complementary to cover energy supply better at those times, for example, VPP formed of photovoltaics (PVs) generates energy during the day and tidal energy may be available at night, while wind energy providers whose turbines are located in the same region will generate energy at the same time and hence be less complementary. In turn, if new actors become better partners due to changes in the environment (more wind blows at night resulting in higher predicted wind energy production than tidal or more EVs converge to a specific region due to a social event, resulting in more storage being available), then some of them might decide to leave their current VPP and form a new one (for example, PV owners may be better storing their excess energy during the day in the EVs to be able to supply at night rather than collaborate with a tidal energy provider. Thus, decentralized coordination algorithms and strategies are more suitable for controlling VPP participants to come to the most efficient arrangements within a reasonable time. A fully distributed energy sharing based on multiagent is described in [16]. It defines a sharing energy strategy based on direct 
interaction between neighboring DERSs, the decisions are made locally using one-to-one communication on the neighborhood area network. The work in [17] focuses on ancillary service coalitions for reactive power contributions. It proposes a distributed control method to launch products of self-organized coalitions of small active units in a power grid at markets for trading active power as well as ancillary services. It consists of the integration of grid restrictions into proactive scheduling of active power with provision of ancillary services, and additionally provides reactive scheduling of active power. The development of VPP integrating DERs and DR relies on the implementation of AMI, ICTs and Network Automation [13]. The VPP deploys an AMI to allow for bidirectional flow of information between consumers, providers and grid devices part of the VPP such as smart meters and sensor-based controllers of power system equipment.

The enrollment of prosumers in VPPs relies on the first movers reported satisfaction or disillusion. VPP reported benefits are likely to draw more participants into VPP. Thus, a VPP could start with a limited number of DERs then it rapidly scale to to thousands of prosumers. Decentralized control or fully distributed control hold much promise regarding managing a dynamic VPP. Moreover, coordination between DER for number of ancillary services for reactive power management is critical for higher integration of DERs within a powergrid [18]. To support these VPPs and the different associated services, the adopted ICT should be scalable, rapidly deployable and capable of peer to peer communication between DERs to allow for flexible smart distributed control schemes. Wireless communication technology is a good candidate for this AMI network. Indeed, Wireless networks have low installation costs and can rapidly be deployed even over wide areas with data rates and network capacities comparable to wired networks ones. Scalable and energy efficient routing protocols are enablers for these networks in VPP context.

\section{ICT for VPP}

\subsection{ISO/OSI model}

Communication neworks products and applications are usually referenced to the ISO/OSI model. This model represents a layer model which defines the process of communication between two endpoints in a network. As presented in Fig. 5, the OSI model is a networking framework composed of seven layers : application layer, presentation layer, session layer, transport layer, network 
layer, data-link layer and physical layer. Each of these layers relies on the layers below it to provide supporting capabilities and performs support to the layers above it [19].

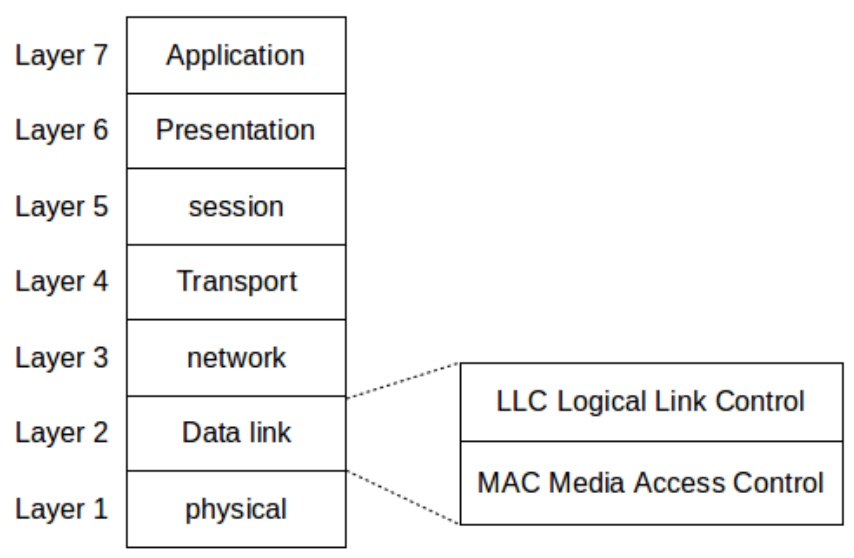

Fig. 5: OSI model

In order to describe how data is transmitted over the network, we consider an example of two endpoints communicating through a physical media, a data packet need to be sent from the sender to the receiver. As shown in Fig. 6, the data is passed from one layer to the next, starting at the application layer of the sender, then proceeding to the bottom layer, over the channel to the receiver and back up the hierarchy. In its way down, address and other headers fields are added to the data packet at each layer, and then removed on the way up.

\subsection{MAC layer}

MAC layer, a sub-layer of the data-link layer, is responsible for Channel access policies, scheduling, buffer management and error control [20]. It aims at moderating access to reduce collisions while facilitating channel reuse and fairness.

Many MAC protocols were proposed for SG such as IEEE 802.11 [21] and IEEE 802.15.4 [22]. IEEE 802.15.4 has been mainly standardized for low power and low data rate communications between devices, which is by contrast to IEEE 802.11 standard developed mainly for end users communications [23]. IEEE 802.15.4 is, then, a strong candidate for machine-to-machine 


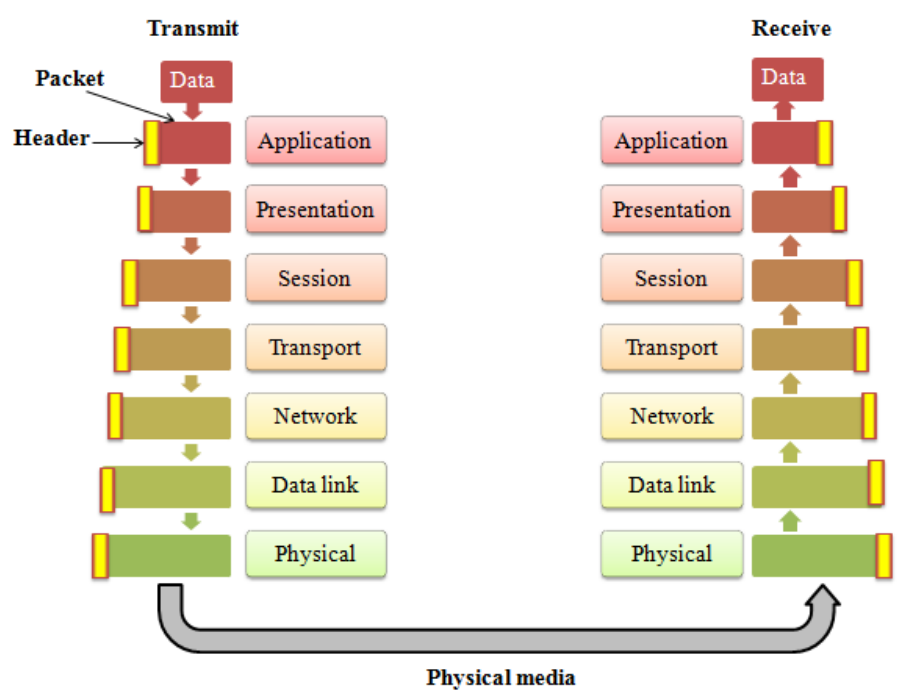

Fig. 6: inter-networking basics - OSI model

applications such as smart grids [23].

A performance evaluation of several MAC protocols is provided in [22]. It proved that IEEE 802.15.4 outperforms other MAC protocols, including IEEE 802.11, in terms of energy conception and packet delivery rate.

For VPP, the ICT should support point-to-point communications. The delivery rate is a critical requirements for $\mathrm{SG}$ communications in general, and VPP in particular. Thus, we will consider IEEE 802.15.4 standard as a MAC layer protocol in the numerical simulation conducted for VPP communication control in this work.

\subsection{Network Layer}

Routing protocols, defined at the network level, are responsible for constructing paths used to route packets between different network entities.

Several routing protocols have been proposed to be used for communications within SG [24]. The routing protocol for Low power and Lossy Networks (LLNs) RPL, designed by the IETF Routing Over Low power and Lossy networks (ROLL) Working Group, is the most most promising routing protocol for SG communication in distribution The routing protocol for low-power and lossy networks (RPL) and geographic routing have been identified as the most promising routing protocol for NANs 
RPL, originally designed by the IETF ROLL Working Group for low-power and lossy networks (LLNs), was proposed for AMI communications [25]. RPL constructs a tree-based routing hierarchy, called destination-oriented directed acyclic graph (DODAG), that maintains routes between smart meters and a DAP.

RPL consists of maintaining network state information using one or more directed acyclic graphs (DAGs) [26]. A DAG is a directed graph wherein all edges are oriented in such way that no cycle exists [27]. Each DAG has a root node and client nodes. A rank, calculated using an objective function, is assigned to each client node in the DAG. The rank monotonically increases in the downward direction so the root has the lowest rank. The objective function is defined using various link and node metrics and constraints to compute the best path.

To create a DAG, RPL uses a set of internet control message protocol (ICMP)v6 messages to exchange graph information. The DAG creation starts by the root sending a control packet called DIO (DAG information object packet). A DIO message contains information about the DAG such as the DAGID used to identify the DAG as sourced from the DAG root; rank information used by nodes to determine their positions in the DAG relative to each other; objective function identified by an objective code point (OCP) that specifies the metrics used within the DAG and the method for computing DAG rank.

If a client node receives a DIO message and decides to join the DAG, it should add the DIO sender (the previous node traversed by the DIO) to its parent list, compute its own rank (associated with the parent node) based on information carried in the message (OCP) ant its local state, and broadcast the DIO message with the updated rank information. In case of loosing connectivity, a node will send out a DAG information solicitation (DIS) message and the neighboring nodes that hear it will respond with a DIO message announcing they are part of DAG. Using the constructed DAG, each client node will be able to forward any upward traffic (an upward traffic is a traffic destined to the DAG root) through its parent as the next-hop node.

To create downward routes from the root to a client node, the client node should issue a control message called destination advertisement object (DAO). A DAO packet includes the rank information used by nodes to determine how far away is the destination (in this case the destinations is the client node). The DAO message will be forwarded to the root using the upward path indicated by the DAG, and all the intermediate nodes record the reverse path 
information from the DAO message, after that, a complete downward path is established from the root to the client node.

The RPL is optimized for multipoints-to-point and point-to-multipoints communication schemes [28]. For that reason, it was proposed as a routing solution for AMI networks [26][29] where traffic is limited from smart meters to a data aggregation point DAP, and from a DAP to smart meters. In this context, different implementations (variants) of RPL routing protocol have been proposed in the literature to meet with NAN requirements. The authors of [30] proposed a routing algorithm based on multiple RPL instances with QoS differentiation at network layer in order to account for NAN's applications requirements. In [31], a modified version of RPL is proposed to support multi-gateway AMI network case. In fact, the standard RPL defines an RPL instance with different disjoint DAGs, each one corresponding to a root. For that, the multi-gateway RPL allow a node to join multiple DAGs in order to increase viable routing options [31]. CORPL [27] is another proposed RPL variant for the context of AMI networks, it addresses the problem of instable wireless links and interference effects.

RPL provides only basic support for point-to-point traffic [29]. When a node sends a packet to another node, the packet uses upward route until arriving to a common ancestor at which point it is forwarded in the downward direction to the destination. To conclude, RPL provides an efficient support of dynamic routing metrics and constraints required by the AMI network. However, establishing and maintaining efficient upward and downward routes according to each possible root can result in high overhead specially for large scale networks which may affect the reliability and scalability of the routing solution. Besides, RPL does not provide an optimized point-to-point traffic support. For these reasons, RPL is not suitable for VPP communication case. For these reasons, RPL is not suitable for VPP communication case. In the next section, we will propose the use of geographic routing for AMI network in the context of VPP.

\section{Geographic GReedy routing with ACO based recovery (GRACO) (GRACO)}

\subsection{Geographic routing}

Geographic routing is a class of routing approaches that exploit geographic position in routing decision in stead of topological information. It has been widely used in ad-hoc and wireless sensor networks for its diverse 
advantages. In fact, GR is a localized routing scheme since packet forwarding decision is achieved by using only information about the position of nodes in the vicinity and the position of the destination node, which minimizes significantly communication overhead. Hence, no additional information has to be stored neither on the nodes on the path, nor in the message which makes GR a memory-less and scalable routing [32]. Besides, GR is also distributed, every node performs the same algorithm [32]

As previously discussed, the deployment of a wireless communication network for VPP faces many challenges. First of all, VPP is supposed to support different applications and multiple traffic flows, the communication network should then manage a huge amount of data, thus, the routing solution should deliver the data packets with the minimum overhead possible so it does not affect the network performances. As VPP is a large scale network with highly dynamic topology due to the regular expansions to cover larger areas and additional endpoints, it needs a scalable routing solution. Besides, the communication scheme for VPP should be resilient to outdoors harsh communication environment and instability of wireless links. For these reasons, the simplicity, localized and distributed operation, low overhead, memoryless and scalability features make GR a very good candidate for VPP.

Some research papers investigated the application of geographic routing for AMI networks. Greedy Perimeter Stateless Routing(GPSR) [33] was proposed in this context. [34] studies the feasibility and the performance of GPSR in NAN scenarios. It was proved that GPSR can support different NAN services in terms of latency and reliability. The authors of [35] compared the performances of GPSR to RPL in the case of NAN network. The simulation results proved that RPL outperforms GPSR in terms of transmission reliability and delay.

\subsection{Ant colony Optimization}

ACO is a meta-heuristic used to solve hard combinatorial optimization problems. It was mainly proposed by Dorigo in his thesis [36][37]. ACO approach bio-inspired from ants foraging behaviour. In fact, real ants are able to find the shortest path between their nest and a food source without any visible, central and active coordination mechanisms. They drop pheromones, a natural chemical substance, on the path. The path optimization is achieved by exploiting the pheromone quantity deposited. Then, ants select a path based on the pheromone concentration deposited on the set of paths found. The higher the concentration of pheromone on a path, the greater the prob- 
ability to select it. This indirect communication mechanism is called stigmergy. In addition to that, real ants show an impressive behaviour when finding obstacles on their way. Actually, they are able not only to avoid obstacles, but also to find a shortest path around them. Fig. 7 shows an example of ants avoiding an obstacle and converging to the shortest path bypassing the obstacle. ACO based approaches are very effectively applied

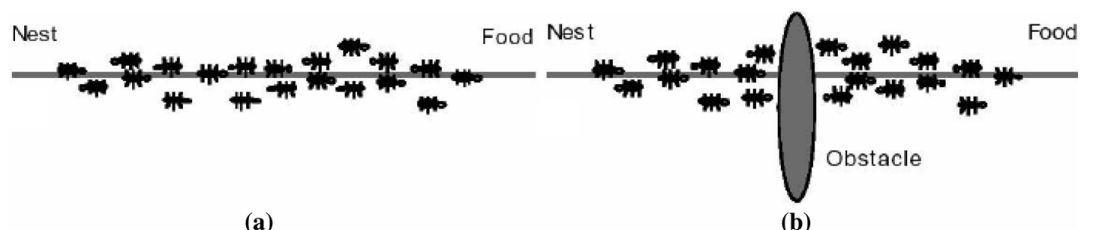

(a)

(b)

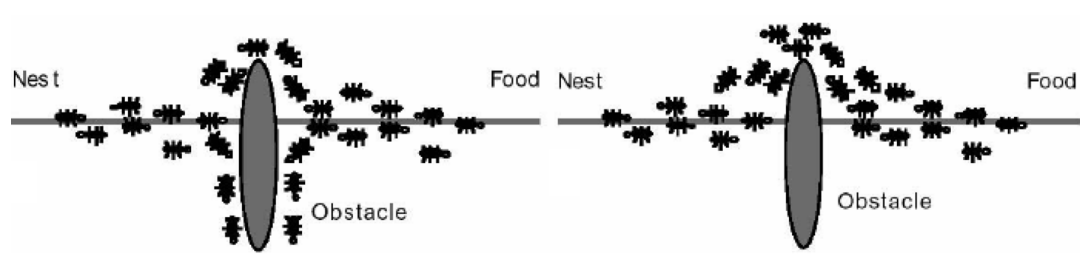

(c)

(d)

Fig. 7: Ants avoiding an obstacle

to NP-hard problems and results in good optimization. Networking field is one of many domains that investigated ACO-approaches to design multiobjective and multi-constraint routing protocol and solve issues like mobility, path optimization, resource utilization and energy awareness [38][39][40] [41]. The ant colony scheme used in this work is not an optimization solution. Although, it is a scheme that is used to find a reliable path for communication between DBRs in a VPP that encounters lost of communciation among them. The ant colony scheme is used in this work becasue it has the ability to adapt rapidly to sudden changes in the network structure caused by physical obstacles or instability of wireless links due to radio frequency propagation and inherent fading.

\subsection{Overview of GRACO}

In [42] , we proposed GRACO a new geographic routing protocol to solve the void problem in wireless sensor networks. GRACO combines geographic greedy forwarding and a novel void avoidance technique based on ACO to effectively deliver data packets. The proposed protocol is the first algorithm 
that uses ACO to assist geographic greedy forwarding. It is fully localized, distributed, scalable, and allows to circonvince voids by providing one or multiple paths around them. Here, we are proposing to apply GRACO in VPP.

In the beginning, GRACO makes the routing decision using geographic greedy forwarding strategy. Greedy forwarding [32] is the simplest implementation of geographic routing. At each step, a node tries to bring the packet closer to the destination, using the euclidean distance as a the progress criteria. Greedy forwarding inherits all the advantages of GR. However it is not always possible, it may lead to a void problem if a packet arrives to a node that has no neighbor closer to destination than itself, the node is, then, stuck and could not progress the packet. GRACO proposes an ACO based strategy to recover from the void problem. The ACO recovery strategy uses two types of ant like packets: Fants (forward ants) to search for a path around the void and Bants (backward ants) to mark the paths found for later use. The Fants will be guided to the direction of the destination using zone concept. Once a Fant arrives to an unstuck node, a node closer to the destination than the stuck node, a Bant is sent to mark the path found by dropping pheromone trails. The recovery is launched with the aim of finding an unstuck node, then, the algorithm switches to greedy forwarding again. The performance of GRACO was compared to the greedy-face-greedy (GFG) routing algorithm. Simulations results presented in [42], shows that GRACO outperforms GFG in term of end-to-end delay, data delivery cost and hop count. Besides, GRACO provides high data delivery rate.

\section{Performance Analysis}

The effectiveness of the proposed routing protocol, reported in section 4 is evaluated through simulation.

\subsection{Simulation Environment}

The simulations are performed using WSNet simulator [43].

In the conducted simulations, we investigated first the performance of GRACO with an ideal MAC layer to evaluate the impact of routing algorithm only in a collision free environment. Then, we checked the performance with more realistic MAC layer, the IEEE 802.15.4 MAC.

The simulations are performed within a region in the downtown of the city of Sfax, Tunisia. We choose to incrementally add nodes to the VPP in order 
to simulate the evolution of the VPP from early stages of a VPP where just a few customers are enrolled in the VPP and the DERs are sparse across the selected region to more advanced stages with denser aggregation of DERs representing a more mature version of the VPP where an important number of prosumers has been attracted by the financial benefits of the DR programs.

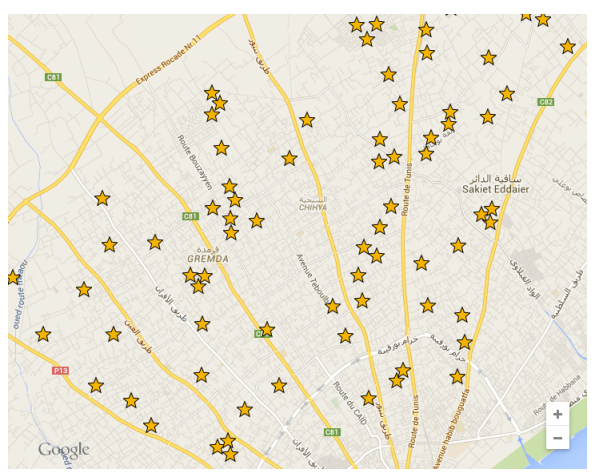

(a)

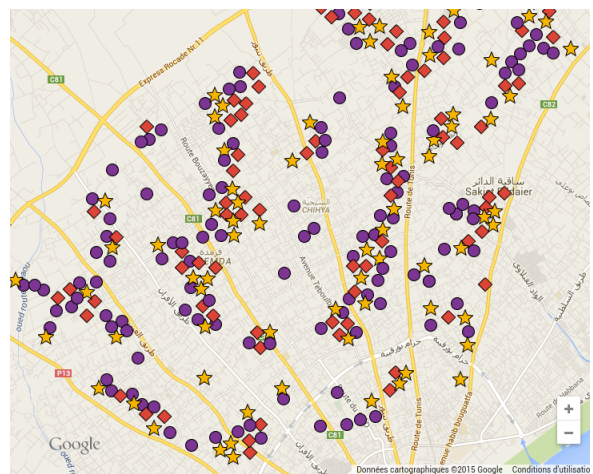

(c)

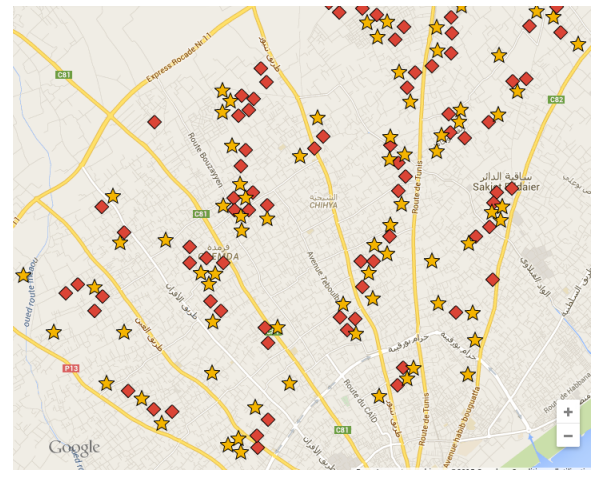

(b)

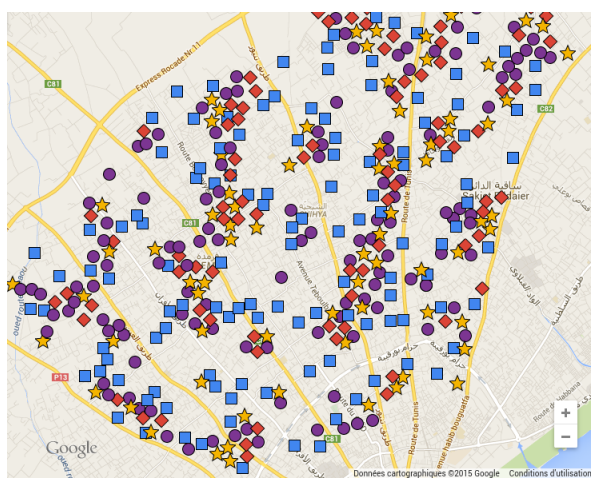

(d)

Fig. 8: Stages of VPP extension within a region in the downtown of the city of Sfax, Tunisia

As more prosumers are enrolled in the VPP, the number of neighbors for one particular connected device, called node degree, will increase. To vary the nodes degree in our simulations, we vary their range from $25 \mathrm{~m}$ to $50 \mathrm{~m}$ by steps of $5 \mathrm{~m}$. Each combination of topology and algorithm is run 50 times. Error bars on curves symbolize a $95 \%$ confidence interval. In order to mea- 


\begin{tabular}{ll} 
Parameter & Value \\
\hline Duration (s) & 300 \\
MAC layer & idealmac and 802.15.4 \\
Interferences & none \\
Density & $10,15,20,25,30,40$
\end{tabular}

Table 1: Simulation parameters

sure the impact of void zones on the algorithm performances, we choose a set of random sources and destinations where there is, necessary, a void to be handled in the routing process. One data packet is sent between a pair of source and destination each 10s.

The performance of the routing protocol is evaluated in terms of the average route length, the end-to-end delay and the data delivery ratio. The route length is the number of hops a data message needs to go from the source to destination. The end-to-end delay is the time interval between a given source sends a packet and the destination receives it. The delivery ratio is the ratio of data packets successfully received by their destinations to all data packets sent by the sources. Table 1 summarizes the simulation parameters.

\subsection{Simulations and Results}

The simulations results show that the more the network is dense the better performs the proposed routing protocol. As the density of the network is increased, shorter paths are found. Specifically, as plotted in Fig. 9, the average route length drops from 59 hops at network density 10 down to 11.94 hops at network density 40.

Fig. 10 shows the end-to-end delay varing with the network density. The average end-to-end delay reduces from $0.781 \mathrm{~ms}$ at a network density 10 to $0.163 \mathrm{~ms}$ at a network density 40 .

The data delivery rate is also improved when increasing network density. In fact, Fig. 11 shows that GRACO provides a 100\% delivery rate with an ideal mac, however, for 802.15.4 MAC, a high reliability of packet transmission is achieved only when the node degree exceed 33 nodes.

To simulate the scalability feature of GRACO, we run simulations on more dense networks with increasing densities starting from 500 nodes per $\mathrm{km}^{2}$ to 4000 nodes per $\mathrm{km}^{2}$. As presented in Fig. 12, the routing protocol GRACO performs poorly in delivering packets at low density networks for both ideal 


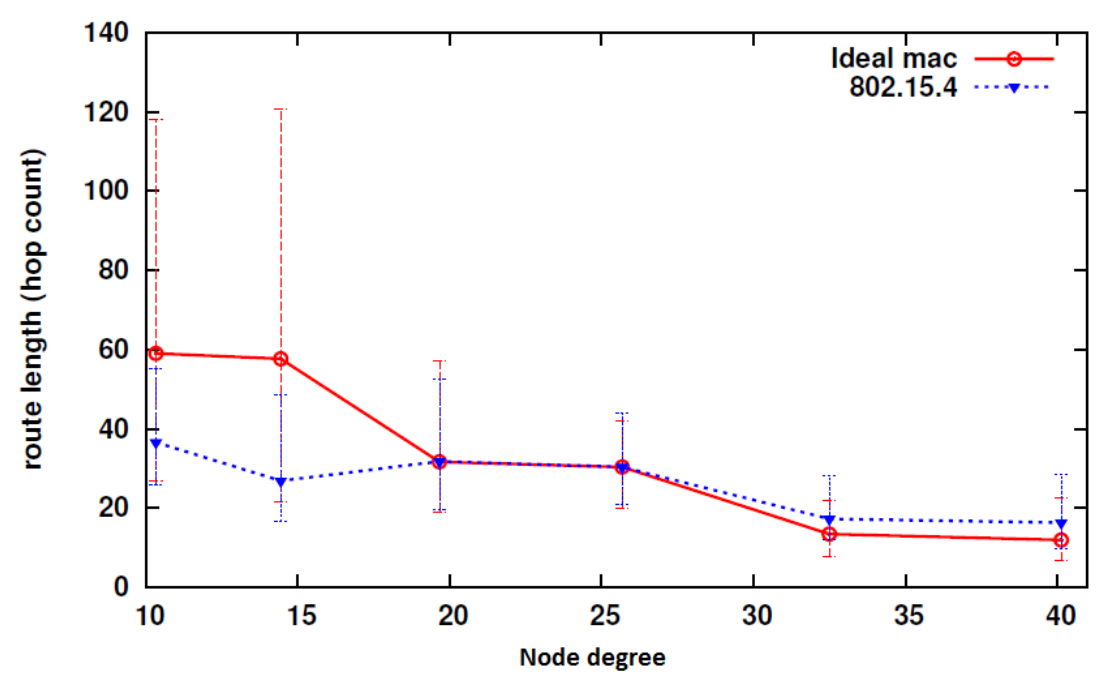

Fig. 9: hop-count

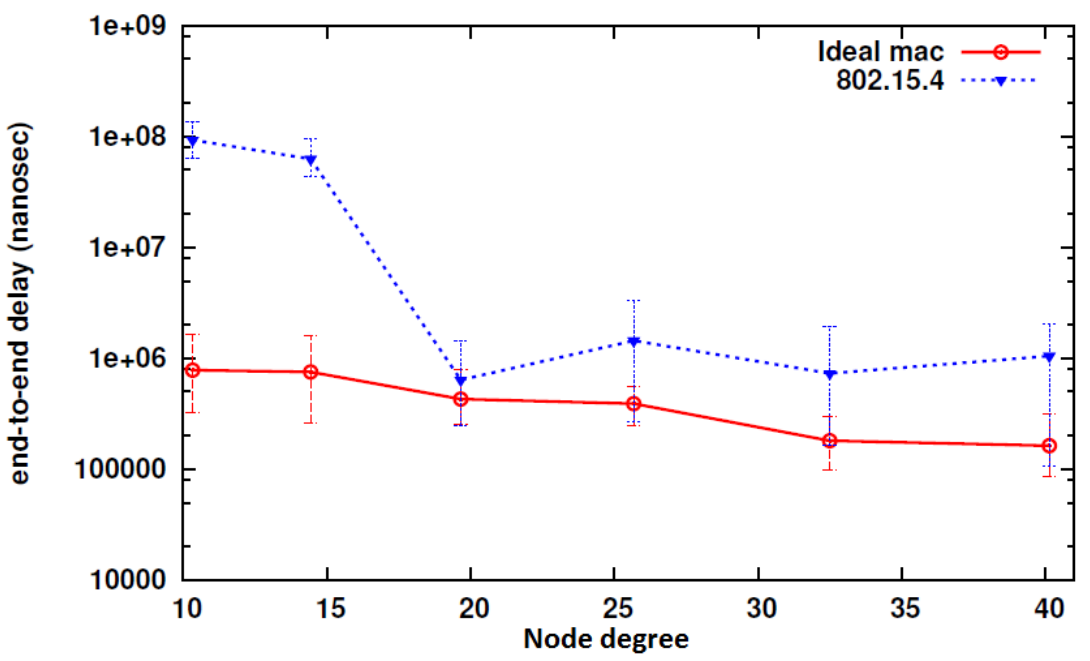

Fig. 10: end-to-end delay

and realistic scenarios. GRACO combined with ideal MAC layer is able to find at least one path between a source and a destination if it exists and delivers $100 \%$ of the data [42]. The only cause that makes GRACO fail to deliver the data in ideal scenario is the nonexistence of a path. Which explain 


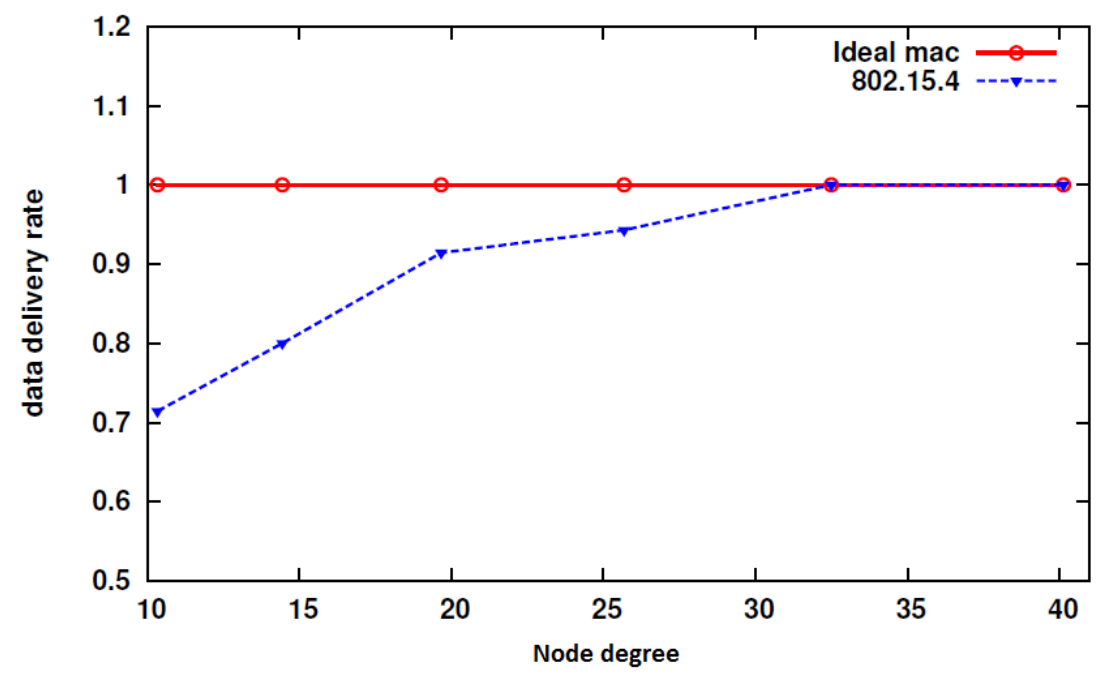

Fig. 11: Data delivery rate

the low delivery rate performed by GRACO with ideal MAC in low density network, $27 \%$ for GRACO with ideal MAC and $25 \%$ for GRACO with IEEE 802.15.4 at density 500 nodes per $\mathrm{km}^{2}$. However, when the networks become denser the connectivity of nodes increases and more paths become available. This explain the increasing delivery rate with the increasing networks size until performing $100 \%$ of delivery rate at density 2000 nodes per $\mathrm{km}^{2}$ in ideal scenario. The performance of GRACO with IEEE 802.15.4 in terms of delivery rate is similar to its performance in ideal scenario. Indeed, using IEEE 802.15.4, the rate of data delivered successfully by GRACO increases with the networks size.

\subsection{Discussion}

When using idea MAC layer, the causes behind packet loss is limited to routing algorithm only. Simulations showed that the routing protocol performs with a $100 \%$ delivery rate in the ideal case, which proves the reliability of the routing protocol. In IEEE 802.15.4 MAC, which is a realistic MAC layer, packet loss may occur due to collision or channel access failure. A collision occurs when two or more nodes transmit the data at the same time. A channel access failure may occur in CSMA/CA mechanism, when a device wants to transmit data, the device waits for a random number of back off 


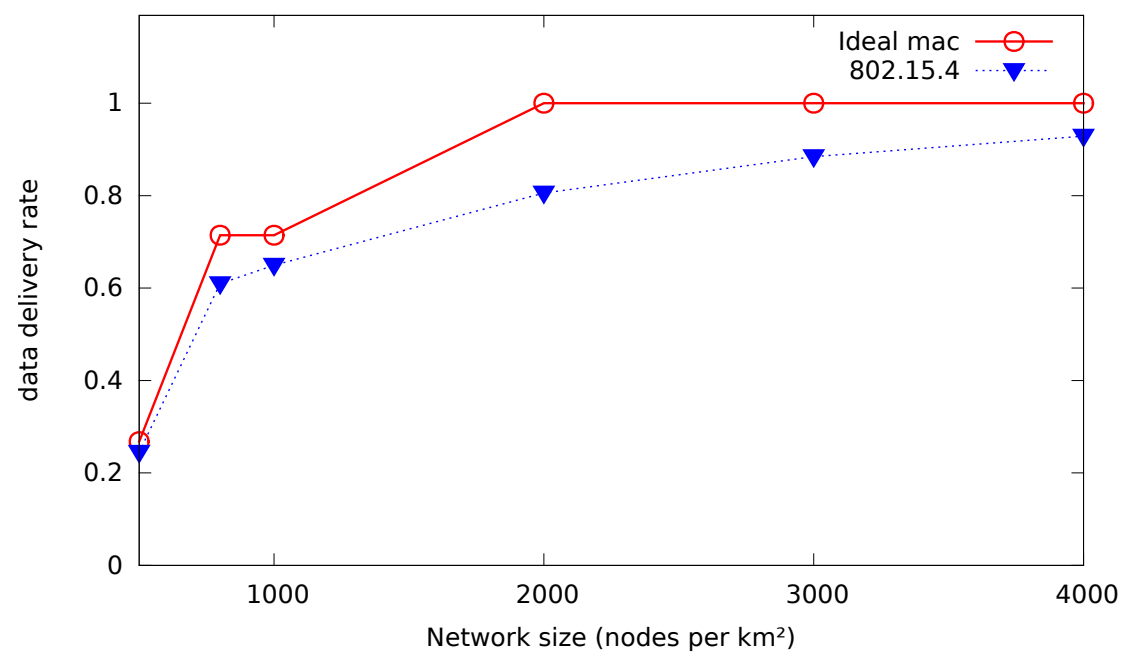

Fig. 12: Data delivery rate

periods before trying to access the channel. The back off time is randomly generated in the interval $\left[0,2^{B E}-1\right][44]$ where $B E$ is the back off exponent, $\operatorname{macMin} B E \leq B E \leq \operatorname{macMax} B E, \operatorname{macMin} B E=3$ and $\operatorname{macMax} B E=5$. The default value of $B E$ is 4 . After waiting a number of back off, the node checks whether the channel is idle. If so, the network device begins to transmit data packet, otherwise, The value of $B E$ is incremented by 1 , the node backs off again with the new value of BE before trying to access the channel. The procedure is repeated until the number of BE exceeds macMaxBE. Similarly, the number of iterations is also limited by NB, which stands for the maximum number of back offs before declaring a channel access failure [44]. The performances of GRACO in the same scenarios but with a realistic mac layer degrades specially in low density networks where alternative paths are hard to find.

\section{Conclusion}

In this paper, we applied ant colony optimization method in conjunction with geographic greedy forwarding mechanism as a routing protocol to support scalable and rapid deployed VPP. The communication requirements of the VPP are discussed in relation with the possible control strategies and 
deployment phases. VPP control strategies could be either centralized, hierarchical or fully distributed. In order to achieve these requirements, our proposed ICT supports multipoint-to-point, point-to-multipoint and pointto-point communications. Typically, VPPs are highly dynamic and enrollment or disenrollment of prosumers in the network could be based on different factors such as economical satisfaction or power needs. As a result, the used ICT should seamlessly scale and adapt rapidly to such dynamic topology. The discussed wireless sensor network technology in this work supports aggregation of geographically sparse DERs into a single VPP. The proposed geographical communication routing protocol, namely GRACO, deliver reliably data between different entities of a VPP. The GRACO combines geographic greedy mode and recovery mode to effectively deliver data packets. Geographic routing is characterized by its simplicity, localized and distributed operation, low overhead, memory-less and scalability features compared to other topological information based routing. Thus, the GRACO inherits the advantages of geographic routing used in the greedy mode. While, in the recovery mode, the GRACO uses an ant colony inspired void handling techniques that adaptively adjust the routing mechanism to avoid blocking situations resulted by sparse deployment of communicating devices, physical obstacles or instability of wireless links especially in urban environments. Hence, it provides scalability and self-healing features to accommodate the dynamic nature of the VPP. In addition to that, the proposed GRACO is flexible enough to support all kinds of communication types; in particular, it ensures point-to-point communication. It allows versatile control strategies for the VPP such as fully distributed control that overcomes challenges of centralized control. Simulations results presented demonstrate that the delivery rate of the GRACO algorithm is within the VPP communication requirements. The simulations showed also that the end-to-end delay decreases with increasing network density or VPP size. This is consistent with the requirements of large DERs communication traffic within large neighborhood with best control strategies decisions.

\section{Acknowledgments}

This work has been partially supported by a grant for the CPER CIA and the MESRST TIC. 


\section{References}

[1] B. Davito, H. Tai, and R. Uhlaner. The smart grid and the promise of demand-side management. McKinsey on Smart Grid, pages 38-44, 2010 .

[2] G. Strbac. Demand side management: Benefits and challenges. Energy policy, 36(12):4419-4426, 2008.

[3] AJ D. Rathnayaka, V. M Potdar, and S. J Kuruppu. An innovative approach to manage prosumers in smart grid. In Sustainable Technologies (WCST), 2011 World Congress on, pages 141-146. IEEE, 2011.

[4] D. Popa, J. Jetcheva, N. Dejean, R. Salazar, and J. Hui. Applicability statement for the routing protocol for low power and lossy networks $(\mathrm{rpl})$ in ami networks. Internet draft, 2011.

[5] M. Rodríguez-Molina, J.and Martínez-Núñez, J.-F. Martínez, and W. Pérez-Aguiar. Business models in the smart grid: Challenges, opportunities and proposals for prosumer profitability. Energies, 7(9):61426171, 2014.

[6] M. Svetina and P. Nemcek. Virtual power plants and utilities. Metering International, (3):133, 2011.

[7] U.S. Department of Energy Aaron Zurborg. Unlocking customer value: The virtual power plant. worldPower, 2010.

[8] E. Mashhour and S. M. Moghaddas-Tafreshi. Bidding strategy of virtual power plant for participating in energy and spinning reserve marketspart i: Problem formulation. Power Systems, IEEE Transactions on, 26(2):949-956, 2011.

[9] E. Mashhour and S. M. Moghaddas-Tafreshi. Bidding strategy of virtual power plant for participating in energy and spinning reserve marketspart ii: Numerical analysis. Power Systems, IEEE Transactions on, 26(2):957-964, 2011.

[10] G. Chalkiadakis, Va. Robu, R. Kota, Al. Rogers, and N. R Jennings. Cooperatives of distributed energy resources for efficient virtual power plants. In The 10th International Conference on Autonomous Agents 
and Multiagent Systems-Volume 2, pages 787-794. International Foundation for Autonomous Agents and Multiagent Systems, 2011.

[11] R. Vasirani, M.and Kota, R. LG Cavalcante, S. Ossowski, and N. R Jennings. An agent-based approach to virtual power plants of wind power generators and electric vehicles. Smart Grid, IEEE Transactions on, 4(3):1314-1322, 2013.

[12] D. Pudjianto, C. Ramsay, and G. Strbac. Virtual power plant and system integration of distributed energy resources. Renewable power generation, IET, 1(1):10-16, 2007.

[13] N. Etherden, M. H. Bollen, and J. Lundkvist. Communication requirements of a virtual power plant using iec 61850 to provide grid services. In Smart Grid Communications (SmartGridComm), IEEE International Conference on, 2013.

[14] H. Yang, D. Yi, J. Zhao, and Z. Dong. Distributed optimal dispatch of virtual power plant via limited communication. Power Systems, IEEE Transactions on, 28(3):3511-3512, Aug 2013.

[15] S. D Ramchurn, P. Vytelingum, A. Rogers, and N. R Jennings. Putting the 'smarts' into the smart grid: a grand challenge for artificial intelligence. Communications of the ACM, 55(4):86-97, 2012.

[16] S. Kahrobaee, R. A Rajabzadeh, L.-K. Soh, and S. Asgarpoor. Multiagent study of smart grid customers with neighborhood electricity trading. Electric Power Systems Research, 111:123-132, 2014.

[17] A. Niese, S. Lehnhoff, M. Tröschel, M. Uslar, C. Wissing, H J. Appelrath, and M. Sonnenschein. Market-based self-organized provision of active power and ancillary services: An agent-based approach for smart distribution grids. In Complexity in Engineering (COMPENG), 2012, pages 1-5. IEEE, 2012.

[18] M. H Bollen and F. Hassan. Integration of distributed generation in the power system, volume 80. John Wiley \& Sons, 2011.

[19] P. Simoneau. The OSI model: understanding the seven layers of computer networks. Global Knowledge Training LLC, 2006. 
[20] A. A. A. Alkhatib and G. S. Baicher. Wireless sensor network architecture. In International conference on computer networks and communication systems (CNCS 2012) IPCSIT, volume 35, pages 11-15, 2012.

[21] NIST Priority Action Plan. 2-guidelines for assessing wireless standards for smart grid applications. National Institude of Standards and Technology Std, 2011.

[22] M. Yigit, E. A. Yoney, and V C. Gungor. Performance of mac protocols for wireless sensor networks in harsh smart grid environment. In Communications and Networking (BlackSeaCom), 2013 First International Black Sea Conference on, pages 50-53. IEEE, 2013.

[23] V.B. Misic and J. Misic. Machine-to-machine communications : architectures, technology, standards, and applications. CRC Press, Boca Raton, 2015.

[24] N. Saputro, K. Akkaya, and S. Uludag. A survey of routing protocols for smart grid communications. Computer Networks, 56(11):2742-2771, 2012 .

[25] Nancy Cam-Winget, Laurent Toutain, Jonathan Hui, Daniel Popa, Matthew Gillmore, and Kazuya Monden. Applicability statement for the routing protocol for low power and lossy networks (rpl) in ami networks. 2015.

[26] D. Wang, Z. Tao, J. Zhang, and A.A. Abouzeid. Rpl based routing for advanced metering infrastructure in smart grid. In Communications Workshops (ICC), IEEE International Conference on, pages 1-6, May 2010 .

[27] A. Aijaz, H. Su, and A.-H. Aghvami. Corpl: A routing protocol for cognitive radio enabled ami networks. Smart Grid, IEEE Transactions on, 6(1):477-485, Jan 2015.

[28] G. Tuna, V. C. Gungor, and K. Gulez. Wireless sensor networks for smart grid applications: a case study on link reliability and node lifetime evaluations in power distribution systems. International Journal of Distributed Sensor Networks, 2013, 2013. 
[29] E. Ancillotti, R. Bruno, and M. Conti. The role of the rpl routing protocol for smart grid communications. Communications Magazine, IEEE, 51(1):75-83, January 2013.

[30] G. Rajalingham, Y. Gao, Q.-D. Ho, and T. Le-Ngoc. Quality of service differentiation for smart grid neighbor area networks through multiple rpl instances. In Proceedings of the 10th ACM Symposium on QoS and Security for Wireless and Mobile Networks, Q2SWinet '14, pages 17-24, New York, NY, USA, 2014. ACM.

[31] P. Thulasiraman. Rpl routing for multigateway ami networks under interference constraints. In Communications (ICC), IEEE International Conference on, pages 4477-4482, June 2013.

[32] N. Mitton, T. Razafindralambo, and D. Simplot-Ryl. Position-based routing in wireless ad hoc and sensor networks. In Theoretical Aspects of Distributed Computing in Sensor Networks. Springer, 2010.

[33] B. Karp and H.-T. Kung. Gpsr: Greedy perimeter stateless routing for wireless networks. In Proceedings of the 6th annual international conference on Mobile computing and networking, pages 243-254. ACM, 2000 .

[34] Q. Ho, G. Rajalingham, and T. Le-Ngoc. Performance and applicability of geographic-based routing in smart grid's neighbor area networks. In Advanced Technologies for Communications (ATC), 2013 International Conference on, pages 215-219. IEEE, 2013.

[35] Q. Ho, Y. Gao, G. Rajalingham, and T. Le-Ngoc. Performance and applicability of candidate routing protocols for smart grid's wireless mesh neighbor area networks. In Communications (ICC), 2014 IEEE International Conference on, pages 3682-3687, June 2014.

[36] M. Dorigo and G. Di Caro. The ant colony optimization meta-heuristic. In New Ideas in optimisation. (D. Corne, M. Dorigo, F. Glover, eds.) McGraw-Hill, 1999.

[37] M. Dorigo and T. Stützle. The ant colony optimization metaheuristic: Algorithms, applications, and advances. In Handbook of Metaheuristics. Springer US, 2003. 
[38] A. M. Zungeru, L.-M. Ang, and K. P. Seng. Classical and swarm intelligence based routing protocols for wireless sensor networks: A survey and comparison. Journal of Network and Computer Applications, 35(5):1508-1536, 2012.

[39] V. Uchhula and B. Bhatt. Article:comparison of different ant colony based routing algorithms. IJCA Special Issue on MANETs, 2010.

[40] P. Sutariya, D.and Kamboj. A survey of ant colony based routing algorithms for manet. European Scientific Journal, 9(10), 2014.

[41] R. Huang and X. Guanghui. Swarm intelligence-inspired adaptive routing construction in wsn. In Wireless Comm. Networking and Mobile Computing (WiCOM), 2010.

[42] M. Rekik, N. Mitton, and Z. Chtourou. Geographic greedy routing with aco recovery strategy graco. In Ad Hoc Now, 2015.

[43] Wsnet simulator. http://wsnet.gforge.inria.fr/.

[44] X. Liang and I. Balasingham. Performance analysis of the ieee 802.15. 4 based ecg monitoring network. In Proceedings of the 7th IASTED International Conferences on Wireless and Optical Communications, pages 99-104, 2007. 